\title{
New technologies for high-risk tunnel construction in Guiyang- Guangzhou high-speed railway
}

\author{
Yubao Zhao • Shougen Chen • Xinrong Tan • \\ Ma Hui
}

Received: 26 June 2013/Revised: 8 November 2013/Accepted: 13 November 2013/Published online: 13 December 2013

(C) The Author(s) 2013. This article is published with open access at Springerlink.com

\begin{abstract}
Based on the construction of high risk tunnels in Guiguang-Guangzhou high-speed railway, several new technologies were developed for high-risk tunnel construction. First, an integrated advanced geological prediction was developed for tunneling in karst area. Then, a new system of ventilation by involving the dedusting technology was proposed and used in the field, which received a good air quality. Finally, a method to minimize the distance between the working face and the invert installation was proposed by optimizing the invert installation and adopting the micro bench method. Applying the method to the project obtained an excellent result. The achievement obtained for this study would be able to provide a valuable reference to similar projects in the future.
\end{abstract}

Keywords Tunneling engineering - High-speed railway · New technologies · High-risk tunnel

\section{Introduction}

The Guiyang-Guangzhou high-speed railway is built from Guiyang of Guizhou Province to Guangzhou of Guangdong Province, as shown in Fig. 1. The railway has a total length of $857.0 \mathrm{~km}$. It is designed for operation at a maximum

Y. Zhao $\cdot$ S. Chen $(\bowtie) \cdot X$. Tan

MOE Key Laboratory of Transportation Tunnel Engineering,

Southwest Jiaotong University, Chengdu 610031, China

e-mail: csgchen2006@163.com

Y. Zhao

e-mail: ybao.zhao@163.com

M. Hui

China Railway Erju Co. Ltd, Chengdu 610032, China speed of $250 \mathrm{~km} / \mathrm{h}$, while reserves a maximum speed up to $300 \mathrm{~km} / \mathrm{h}$ in the future. All tunnels in the railway are in double-track with a large section of $92 \mathrm{~m}^{2}$ above the rail level.

The Construction Contract I (GGTJ1) of Guiyang-Guangzhou high-speed railway covers the line from Guiyang to Duyun in Guizhou Province as shown in Fig. 1 [1]. The contract is $69.26 \mathrm{~km}$ long and has 23 tunnels with a total tunnel length of $49.8 \mathrm{~km}$; i.e., the total tunnel length occupies $71.8 \%$ of the whole contract length. Four of the 23 tunnels are classified as high-risk tunnels of Grade I, namely Taiyangzhuang tunnel, Youzhushan tunnel, Doupengshan tunnel, and Tuanzhai tunnel. As the tunnels are located in karst area, the main risk during the tunneling is potential water/mud bursts. The water/mud bursts could cause a serious loss in tunneling. An example is the water/mud burst occurred in Yeshanguan tunnel of Yiwan railway on 5th August 2007, which killed ten workers. Other challenges of this project include high gas content, high standard of construction quality, and tight period requirement.

The major difficulties during the tunneling include [2]: (1) Geological prediction Water/mud bursts relate closely to geological conditions, especially the caves in rock; a precise prediction of the karst caves, therefore, becomes one of the main concerns in order to avoid water/mud bursts. (2) Air quality assurance The drill and blast method with trackless transportation is adopted, which is very difficult to assure the air quality during the tunneling. An effective way for ventilation and air purification is required. (3) Invert construction According to the Chinese specification, a maximum distance of $35 \mathrm{~m}$ between working face and invert installation is required for rock class IV and V. As the full face excavation method cannot assure the tunnel stability, the bench method is often used, but adopting the traditional bench method would be very 


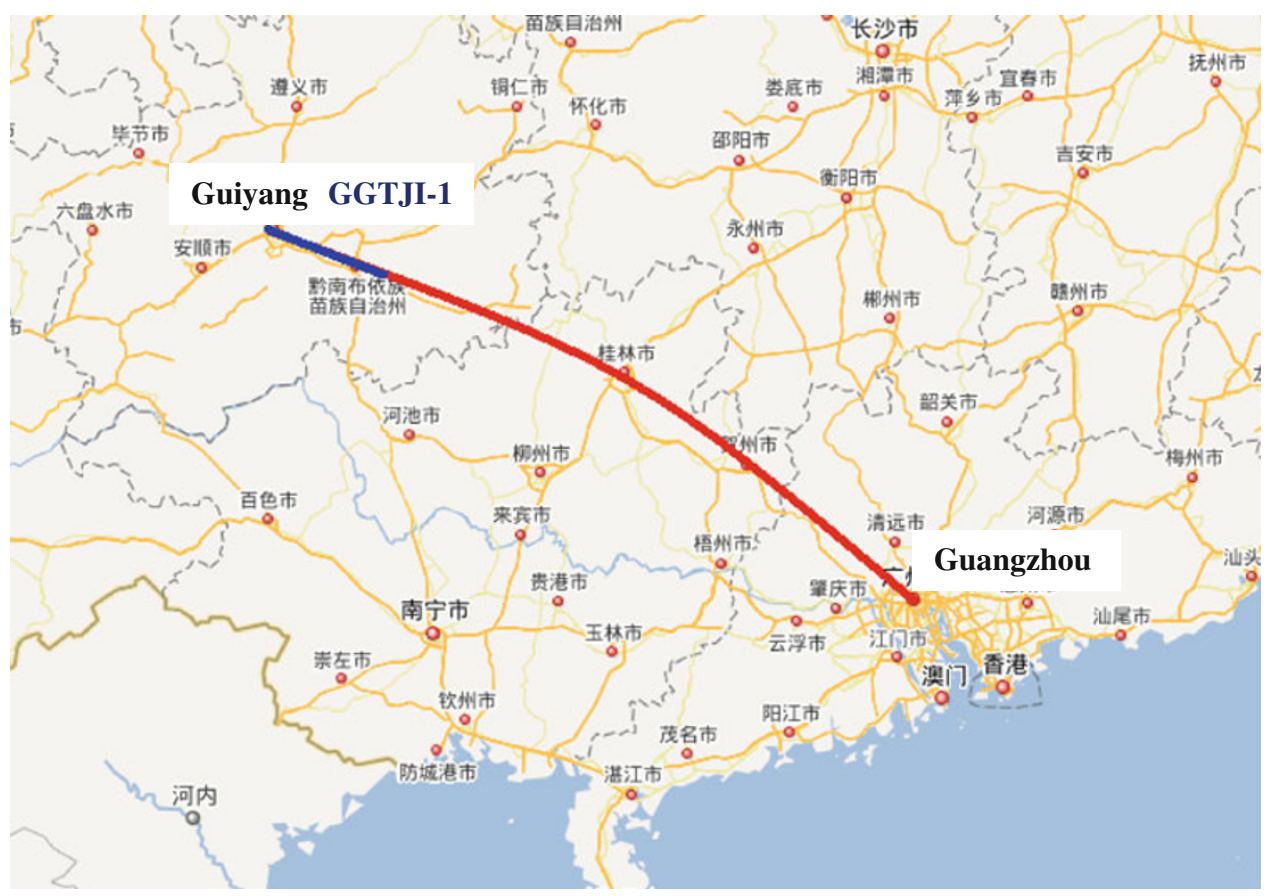

Fig. 1 The Guiyang-Guangzhou high-speed railway

difficult to meet the maximum distance requirement. Thus efforts must be made to solve these problems.

This study is to investigate the key technologies of highrisk tunnel construction in Guiyang-Guangzhou high-speed railway. An integrated advanced geological prediction was developed for tunneling in karst area. Then, a new ventilation system involving dedusting technology was proposed and used in the field, which received a good air quality. In addition, a method to minimize the distance between the work face and the invert installation was proposed by optimizing the invert installation and adopting the micro bench method. Applying the method to the project obtained an excellent result.

\section{An integrated advanced geological prediction system}

The geology that a tunnel passes through is often so complicated that it is impossible in cost or technology to clearly understand the geological condition before the tunnel excavation. The recently developed advanced geological prediction technology is to predict the geological condition from the tunnel working face during the tunneling. Several methods of geological prediction technologies have been proposed, but it is found that each method has its adaptability [3]. For example, some of them may be more favorite to detect water, but others fractured zones; some may be effective to long distance, but others short distance. As tunneling is a dynamic process, the previous long distance could be a short distance for the current step [4-6].

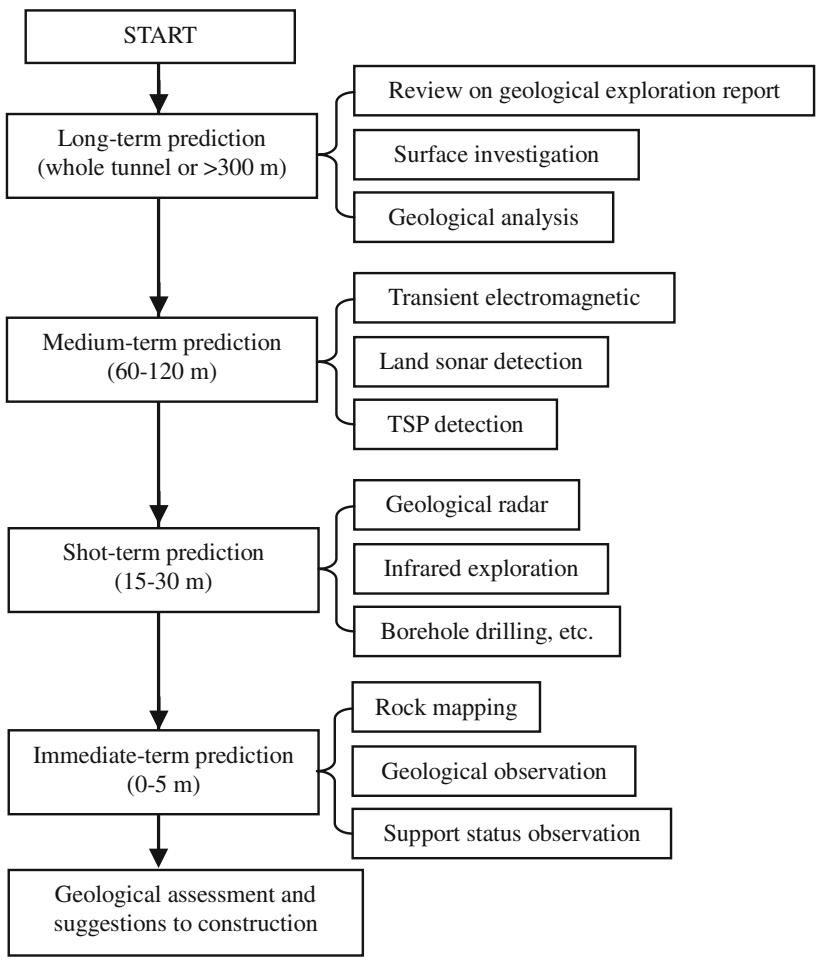

Fig. 2 An integrated geological prediction system

Therefore, an effective geological prediction system should take account of the dynamic tunneling process and the adaptability of each geological detection technology [7, 8].

An integrated geological prediction system was proposed as shown in Fig. 2, in order to obtain a reliable result of 
geological prediction during the tunneling of the present project. The system includes four stages: long-term prediction, medium-term prediction, short-term prediction, and immediate-term prediction. The long-term prediction is to find out the macroscopic geologies such as rock type, strata logging, major fractured zones, and faults for the whole tunnel or a distance longer than $300 \mathrm{~m}$. It can be done by reviewing the geological exploration report, conducting surface investigation, and geological analysis. The mediumterm prediction is to predict the major fractured zones and faults in a more detail, and water condition, up to 60-100 m in front of the working face. It can be done mainly by geophysical prospecting, including transient electromagnetic detection, landsonar detection, or tunneling seismic prediction (TSP). The short-term prediction is to further refine the geology, up to 15-30 $\mathrm{m}$ in front of the working face, based on the results of long-term and medium-term prediction. It can be done by geological radar, infrared exploration, or borehole drilling, etc. The immediate-term is, however, to finalize the geology for the next excavation step by carrying out rock mapping, geological observation, support status observation, etc. Each of the four stages above can provide complement and verification to another in order to achieve a reliable geological prediction result. Based on the result, the geology is assessed, and suggestions can be provided for guiding the tunnel construction. As shown in Fig. 2, different from the existing methods, this geological prediction system integrates multiple technologies newly developed in geophysics, based on the traditional work of rock mapping and geological analysis, and works step by step to provide a more reliable prediction result.

The integrated geological prediction system described above was applied to the geological prediction of Section DK100+750-950 of Doupengshan tunnel in Guiyang-Guangzhou high-speed railway. The tunnel is located at a karst area with major rock of dolomitic limestone, belonging to soluble rock. Based on the review of the geological exploration report, the surface investigation was conducted by checking the topography and geomorphology above the whole tunnel. A creek was found to flow through the section of DK100+750-950. The lowest location of the creek formed the smallest overburden of the tunnel.

As a long-term prediction, a TSP detection was done at the working face of DK100+699, which found that the section between DK100+789-819 would be a fault with fractured rock and caves containing rich water. In order to further make sure the result, another TSP detection was carried out at the working face of DK100+765. The TSP detection result verified the previous result and revealed that unfavorable geology would be more or less at DK100+782-801, DK100+806-810, and DK100_817-819, as shown in Fig. 3, where the marks of triangle, circle, and square represent $\mathrm{P}$-wave, $\mathrm{SH}$-wave, and $\mathrm{SV}$-wave, respectively.
As a short-term prediction, a radar detection was done at the working face of DK100+765, in which five lines were used, as shown in Fig. 4 [9]. The detection result of Line 1 (Fig. 5) shows that there would be four zones with abnormal signals, at DK100+768-770, DK100+774-780, DK100+774-780, and DK100+785-788, respectively.

Finally, rock mapping and geological observation at working face were carried out. It found that water started to be seen at DK100+767, and the water flow gradually became larger along with the tunnel advancing, which verified the above prediction result.

From the geological prediction above, a geological prediction result was finalized as shown in Fig. 6. From the figure, it can be seen that one pipe-shape cave filling with clay and gravels laid through this area from DK100+770-795. Two fractured zones with width of 2-3 m were predicted at around DK100+778 and DK100+780, respectively, warning that a potential water/mud burst would occur if no measure was taken.

Based on the prediction result, the tunneling method was changed to bench method by small advancing rate $(0.5-1.0 \mathrm{~m} /$ round) from full face method to assure the stability of the tunnel. Long drill holes were adopted to detect the water pressure and allow an earlier water leakage to decrease the water pressure. At the same time, grouting was applied to strengthen the rock. By using the above measures, the construction succeeded. After the excavation, the treated cave and fractured zones were clearly observed on site, which in turn verified the geological prediction result. From this study, it was found that the geological prediction in karst strata would focus on caves and water.

\section{A ventilation system by involving dedusting technologies}

The drill and blast method is widely adopted in tunnel construction of high-speed railways. Therefore, improving the air quality in tunnels is critical to keeping a good working environment in tunnels, especially for a long tunnel with a high gas content. The traditional ventilation is to dilute harmful gas and dust. However, it is found that using ventilation only is not effective or costs a lot of electricity [10].

In this project, a ventilation loop distance is longer than $10 \mathrm{~km}$. The traditional ventilation scheme using ventilators only will cost a lot and cannot assure a good air quality. To solve this problem, a dedusting technology is involved in the ventilation scheme, by using a dust collector as shown in Fig. 7.

Selection of dust collector depends much on the dust type, effectiveness, cost, size, maintenance, etc. Beside meeting the requirement of the national work health standard, dust collectors used in tunneling must also be able to 


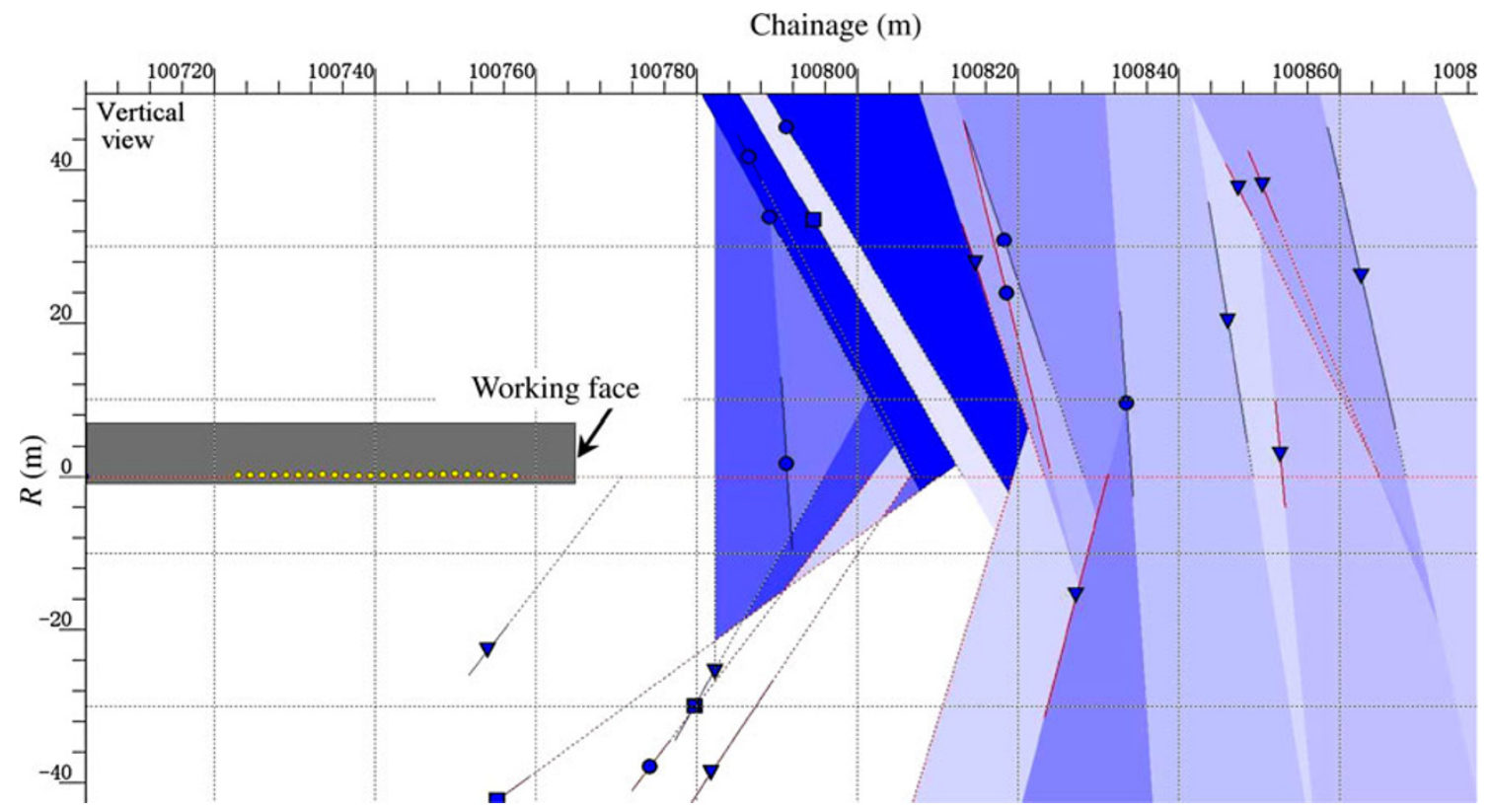

Fig. 3 TSP detection result

collect the dust with a diameter between 0.4 and $0.5 \mu \mathrm{m}$, workable in an environment with relative humidity of $90 \%$ or above, and occupy a smaller space. Three type of dust

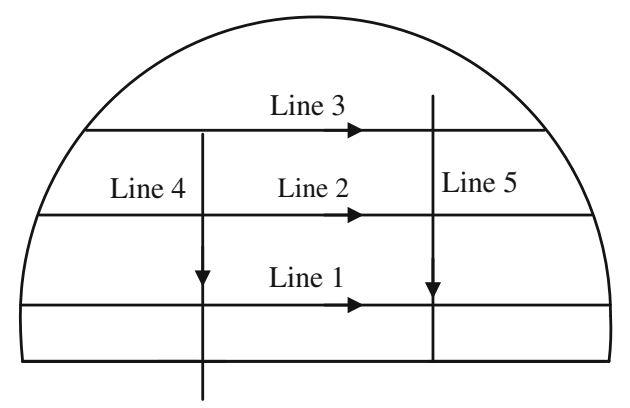

Fig. 4 Layout of radar survey lines and drilling holes collectors meet the above requirements including wet dust collector, bag dust collector, and franklinic dust collector. Compared to the other two types of collectors, the bag dust collector has advantages of high effectiveness, smaller space occupying, higher stability and reliability, lower noise, and easier maintenance. Therefore, bag dust collectors have found wide applications.

A bag dust collector with brand of Chuanshanjia as shown in Fig. 8 was used in this project. It has a smaller size which is only $1 / 5$ of normal bag dust collectors, a power of $15 \mathrm{~kW}$, and costs only about 1,50,000 RMB. The bag dust collector was placed at a certain distance from the blasting in order to protect the machine, and a soft pipe was used to extend the wind entrance to the working face during the use.

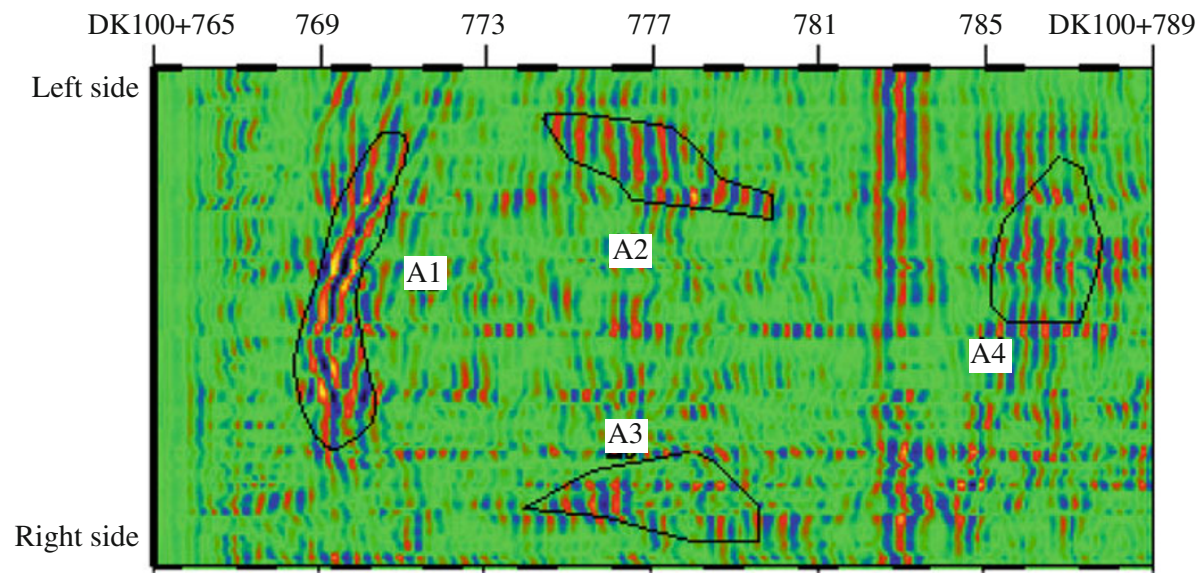

Fig. 5 Geological radar detection result of Line 1 


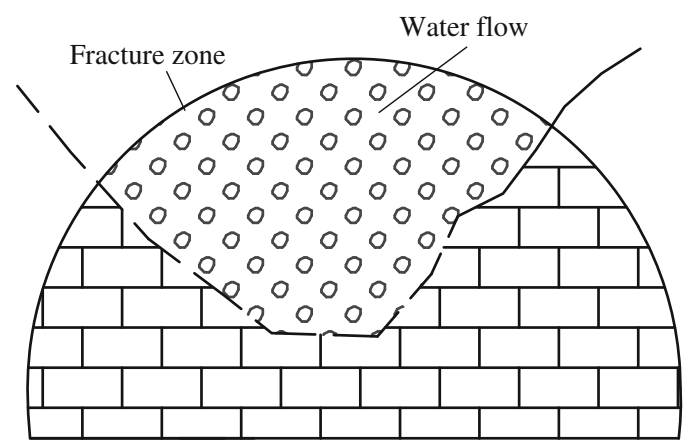

(a)

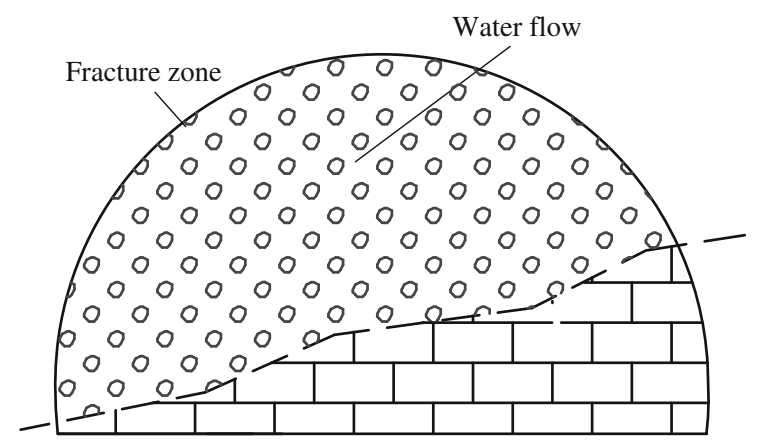

(b)

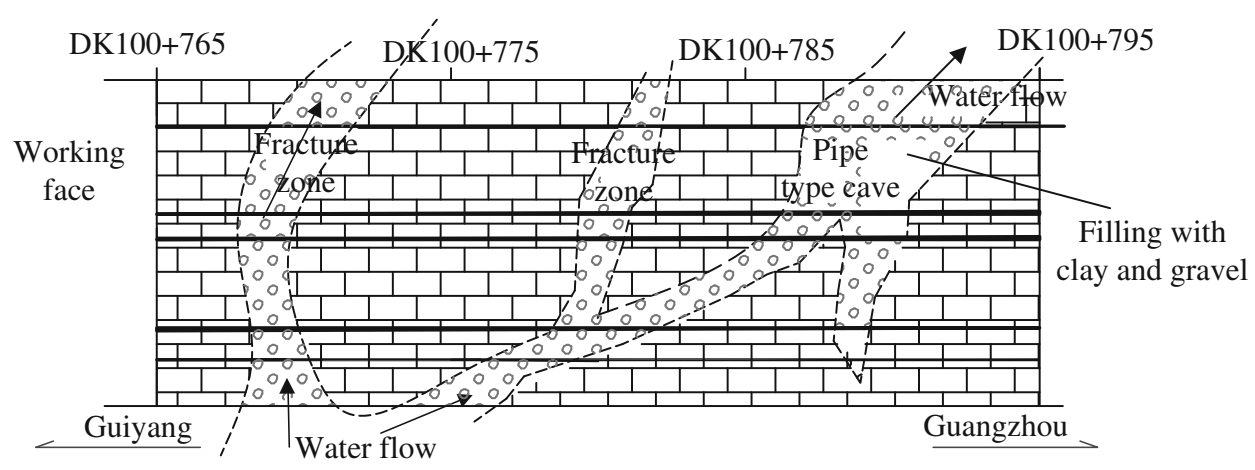

(c)

Fig. 6 Finalized geological prediction result. a Section at D3K100 + 770. b Section at D3K100 + 790. c Longitudinal section

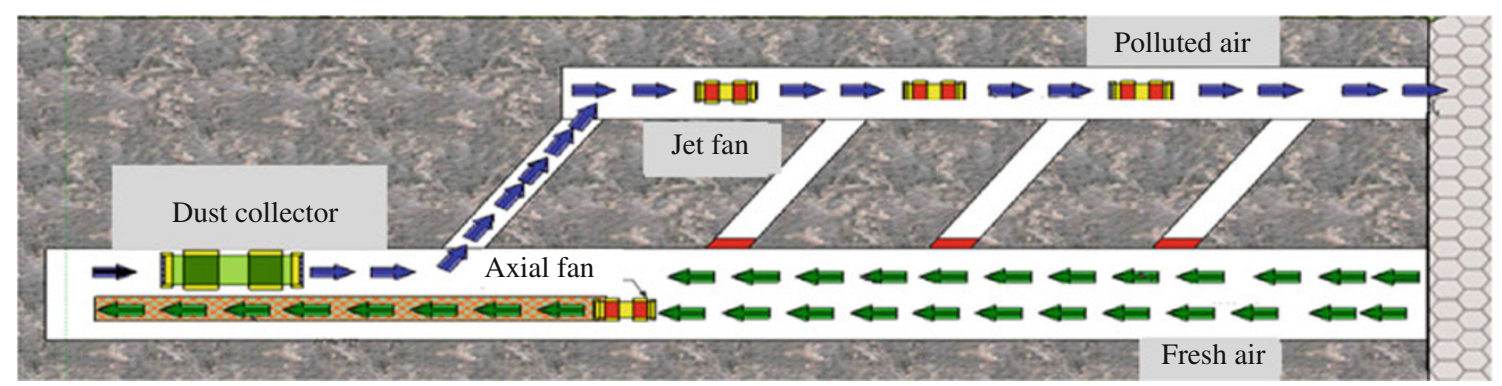

Fig. 7 The ventilation system by involving 1 dust

Site tests indicate that the dust collector could absorb $96 \%$ of the total dust generated by rock blasting. The ventilation system as shown in Fig. 7 was then applied to the project, which received a good result in electricity savings and air quality improvement [11].

\section{Technologies for qualified bench excavations}

Compared with traditional railway tunnels, double-track tunnels in high-speed railways have a big section area in order to minimize the aerodynamic effect. The bench tunneling method is often used to assure the tunneling stability. However, according to the Chinese specification
"Safety Technological Specification of Railway Tunnel Construction (TB10304-2009)," the bench length must be within $50 \mathrm{~m}$ for rock grade IV, and $40 \mathrm{~m}$ for rock grade V. On the other hand, the Chinese Ministry of Railway published "Notice of Further Provisions on Railway Tunnel Design and Construction in Weak Rock and Unfavorable Geology (Railway Construction 2010120)," which requires that the distance from the working face to the invert installation cannot be longer than $35 \mathrm{~m}$ for rock grade IV or worse, in order to strengthen the tunneling safety [12]. It is obvious that the traditional bench methods (long or short) cannot meet the requirement. In order to solve this conflict, effort was made by optimizing the invert installation and applying the micro bench method. 


\subsection{Invert installation optimization}

The advance rate of the invert installation is the most important in the tunnel construction, as it affects greatly the construction of other working procedure such as excavation advance rate, drainage system, and lining. However, in the current railway tunnel construction, due to lack of relevant construction equipment and technologies, it is very difficult to assure a fast invert construction advance rate and achieve an integral invert casting [13, 14]. In addition, with the increase of railway speed and tunnel section area, higher construction quality of tunnels is required in order to assure the tunnel quality and safety. For example, the invert is required to have an integral casting with no construction joints, and the invert casting and back filling must be done separately.

In order to meet the requirement in the invert installation, a rig was developed to achieve a fast invert construction with high quality, as shown in Fig. 9. The rig consists of five parts:

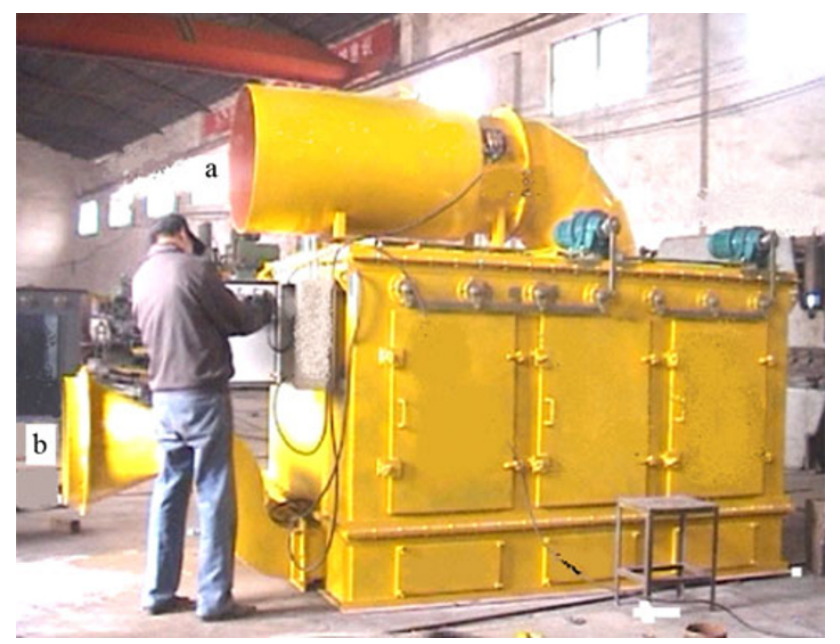

Fig. 8 The dust collector. a Wind entrance. b Wind exit

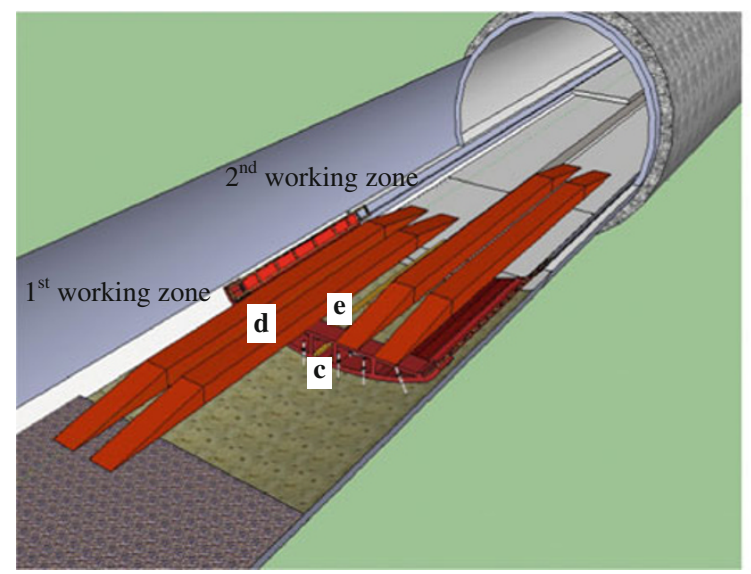

(a) Invert mold frame used to carry out fast positioning and an integral casting with no construction joints. (b) Central drain mold frame used for the concrete casting of central drain. (c) End beam used for positioning and fixing of molds. (d) Trestle bridge used for traffic and acting as a hanging beam for moving molds and end beam. (e) Moving system, a rail system attached to the trestle bridge to move the whole rig. The end beam is to divide the invert construction into two working zones: in front of the end beam is the first working zone to carry out excavation, mucking, and cleaning the bottom, while the second working zone is behind the end beam, which is for carrying out mold installation, mold moving, and concrete casting.

By using the rig above, a new method of invert installation was proposed (Fig. 10). The construction in the two

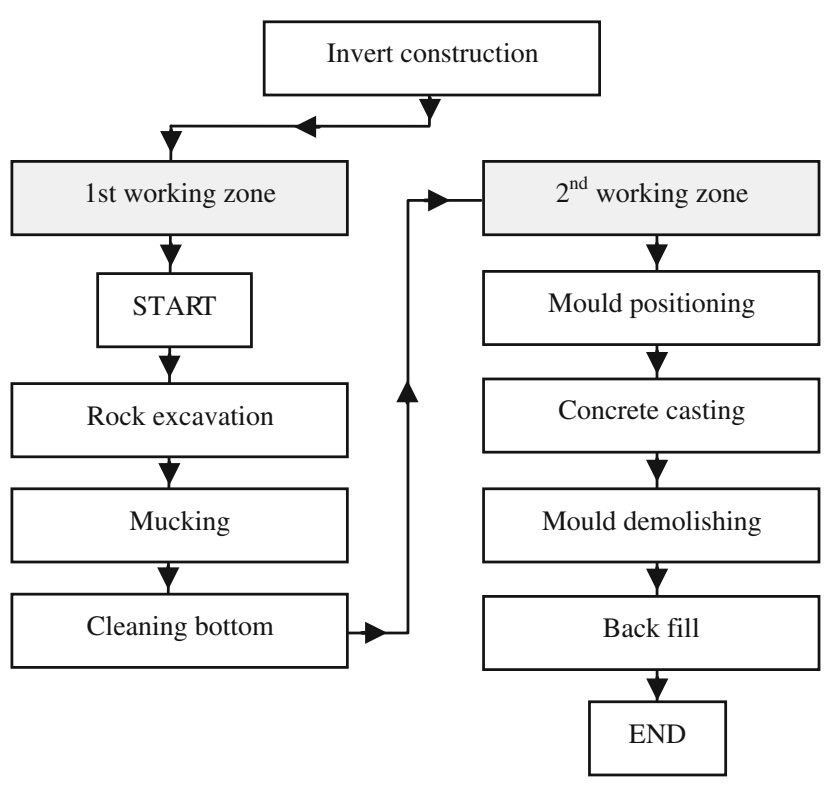

Fig. 10 Flow chart of the new method in invert construction

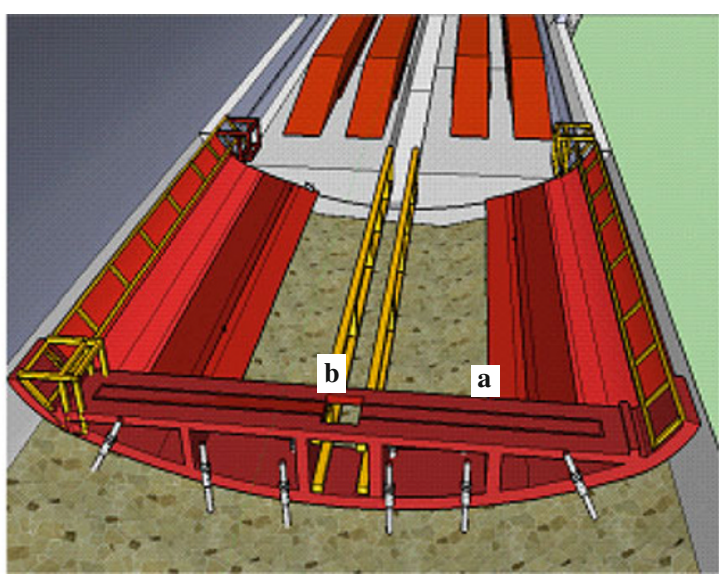

Fig. 9 The rig developed for the invert installation. a Invert mold frame. b Central drain mold frame. c End beam. d Trestle bridge. e Moving system 
working zones was performed in a flow production. Comparing this method with the traditional one in invert construction found that using the new method could achieve a $6 \mathrm{~m}$ invert installation in $19 \mathrm{~h}$, in contrast to $58 \mathrm{~h}$ by using the traditional method; and the cost can be reduced from 225 to $80 \mathrm{RMB} / \mathrm{m}$. In addition, as the whole invert was installed at the same time, a better invert quality can be achieved by the new method.

\subsection{The micro bench method}

To maintain the tunnel stability, a bench length of 3-4 $\mathrm{m}$ in the mirco bench method was designed (Fig. 11). The

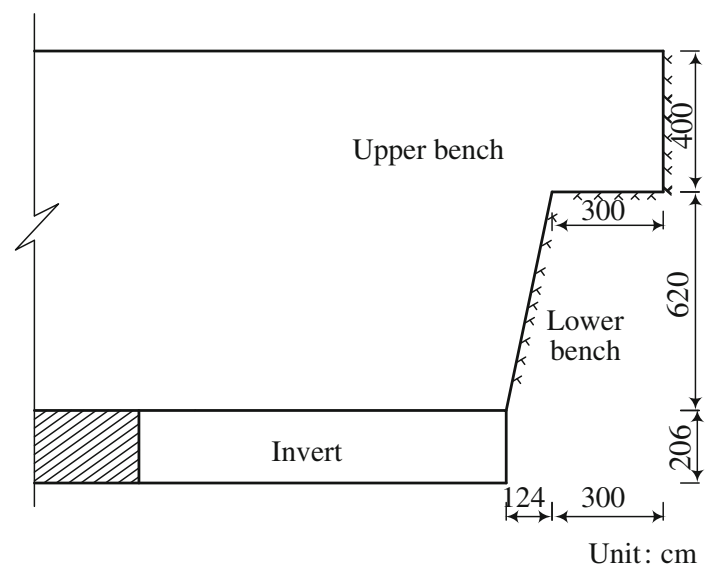

Fig. 11 The micro bench layout

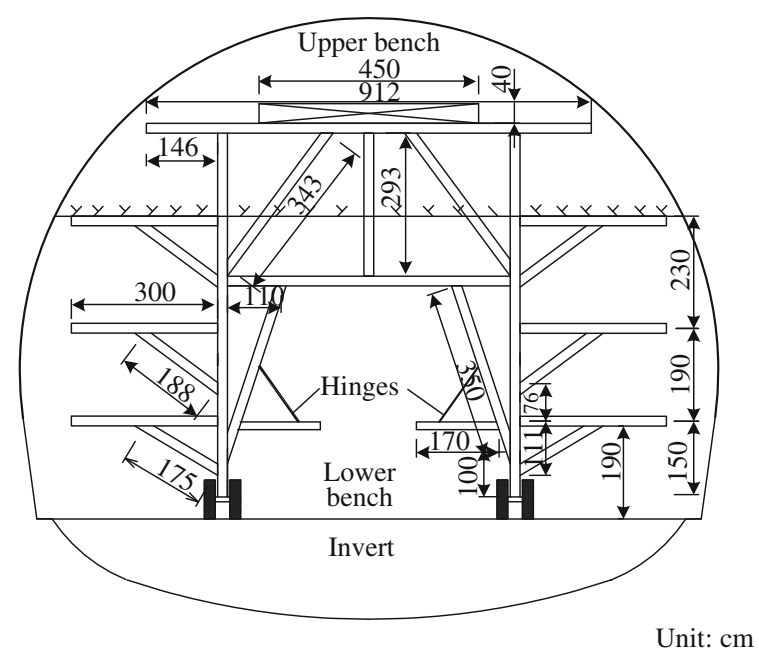
(a)
Unit: $\mathrm{cm}$ excavation height of the tunnels is about $10.2 \mathrm{~m}$. The excavation height at upper bench and lower bench is 4.0 and $6.2 \mathrm{~m}$, respectively. To perform the micro bench excavation, a multi-function platform was innovated by adding a cantilever to the existing rig for the full face excavation as shown in Fig. 12. The forward cantilever can be easily demolished in case of full face excavation for rock grade III [15]. The platform is equipped with four wheels and moves by manual pushing. It will be moved away to a certain distance from the working face in order to protect the platform when blasting.

The micro bench method was designed as shown in Fig. 13. From the figure, it can be seen that the construction cycle includes: (1) set up the platform, (2) drill holes and detonate, (3) mucking at upper bench, (4) support installation at upper bench, (5) mucking and support installation at lower bench, and then turns to the next construction cycle. Through the cycle, a closer sequence of drilling holes, removing mucks, and applying support is achieved, which can be regarded as an evolution from the full face excavation. More importantly, it can increase the efficiency of tunneling in weak rock, provide a bigger space for consequent works, reduce the distance between the working face and the invert/lining to meet the specification requirement, and assure the safety and advance rate of the tunneling. In addition, rock deformation monitoring in site showed that by adopting the micro bench method, the crown settlement and sidewall convergence were reduced compared with the conventional method.

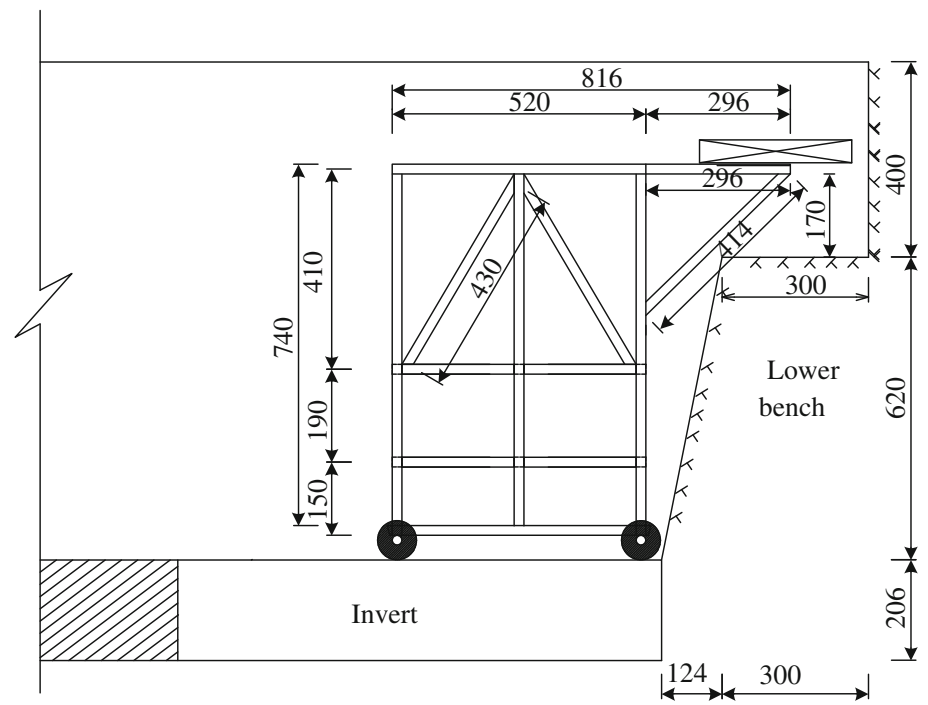

(b)

Fig. 12 The platform for micro bench excavation a Front view. b Side view 


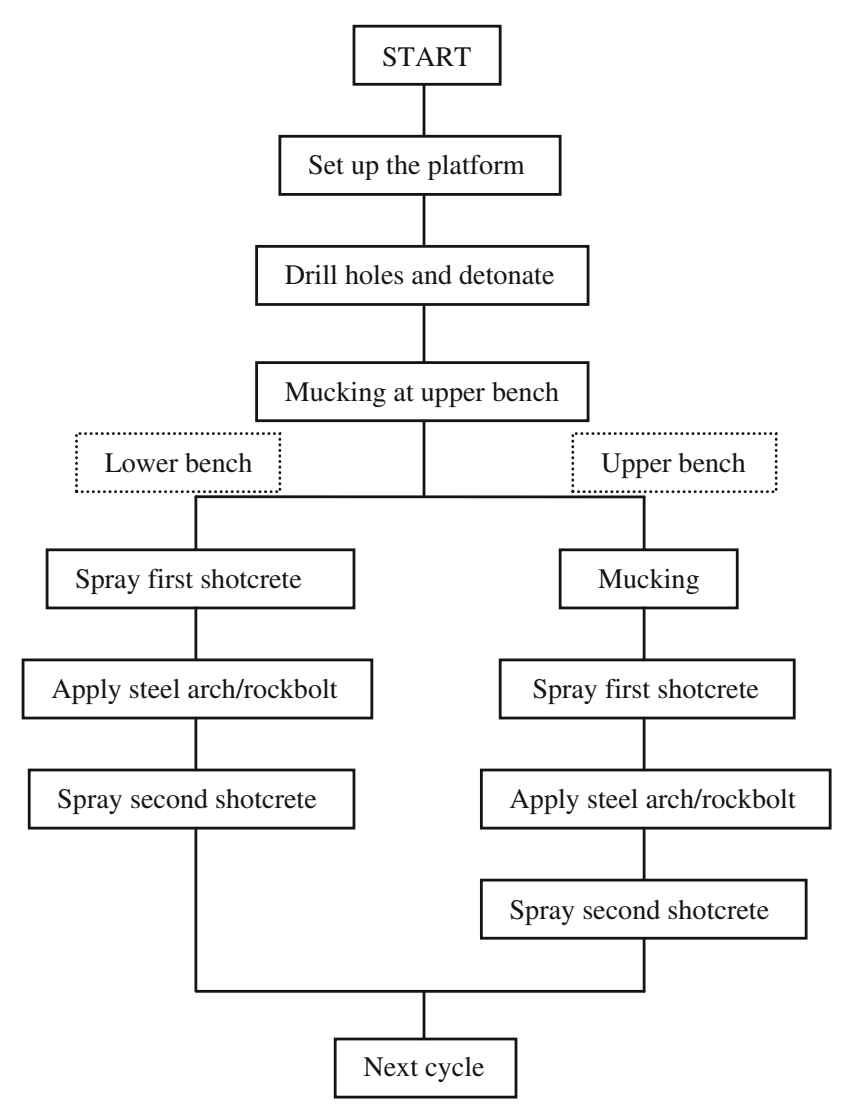

Fig. 13 Flowchart using the micro bench method

\section{Conclusions}

This study investigated new technologies for high-risk tunnel construction in Guiyang-Guangzhou high-speed railway. From this study, conclusions can be drawn as follows:

- An integrated geological prediction system including long-term, medium-term, short-term, and immediateterm predictions was proposed. The application of the system in the field showed that it could significantly increase the accuracy of the geological prediction.

- A ventilation system by involving a duster was proposed. The system was applied to the project and received a good result in electricity savings and air quality improvement.

- By innovating the invert installation rig and the platform for micro bench excavation, the maximum distance required by the specification can be met, a big cost can be saved, and the safety and a fast advance rate of the tunneling can be assured.

- The experience obtained from this study would be able to provide a valuable reference to similar projects in the future.
Open Access This article is distributed under the terms of the Creative Commons Attribution License which permits any use, distribution, and reproduction in any medium, provided the original author(s) and the source are credited.

\section{References}

1. Ma H, Chen SG (2011) A study on construction technology of high-speed railway tunnels with a high risk at karst area and their applications. A project report, China Railway Erju Co. Ltd, Chengdu (in Chinese)

2. Ma H, Liu R, Chen SG et al (2011) Several technological issues to be urgently solved in current railway tunnel construction. Chin J Modern Tunnel Technol 48(5):1-6 (in Chinese)

3. Yan C, Yang J, Chen SG (2008) The application of an integrated geological prediction in Fault F6 of Jinping traffic tunnel. Chin J Highw Tunnel 1:34-38 (in Chinese)

4. Ma H, Chen SG, Tan X (2011) A study on the geological prediction technique for the construction of karst tunnels. Chin J Sichuan Architect 31(2):167-169 (in Chinese)

5. Yang G, Yang L (2006) A study on geological prediction and detection technologies for tunnel construction. Chin J Undergr Space Eng 2(4): 627-630, 645 (in Chinese)

6. Ma H, Chen SG, Tan X et al. (2012) Advanced geological detection for tunneling in karst area. In: ASCE Geotechnical Engineering State of the Art and Practice, San Francisco, 25-29 March 2012

7. Chen SG, Zhang H, Tan X et al (2011) Key technologies for construction of Jinping traffic tunnel with an extremely deep overburden and a high water pressure. J Modern Transp 19(2):94-103

8. Bai B, Zhou J (2001) Advances and applications of ground penetrating radar measuring technology. Chine J Rock Mech Eng 20(4):527-531 (in Chinese)

9. Wu J, Mao H, Ying S et al (2003) Application of ground probing radar to short-term geological forecast for tunnel construction. Chin J Rock Soil Mech 24(S):154-157 (in Chinese)

10. Tan X, Chen SG, Zhang H (2012) Optimization of construction ventilation of long and big tunnels based on the monitoring of air quality in tunnels. Modern Tunnel Technol 49(6):152-157 (in Chinese)

11. Tan X, Chen SG, Ma H (2012) Application of air quality monitoring in long and big tunnels. Chin J Safety Environ 12(6):103-107 (in Chinese)

12. Ma H, Liu R, Chen SG et al (2011) Opinions on the tunneling management in current railways. Chin J Modern Tunnel Technol 48(S):61-65 (in Chinese)

13. Zou C, Shen Y \& Jin Z (2013) Study of bench method's geometric parameters optimization in weak broken wall rock and large cross-section tunnel. Chin J Highw Eng 38(2):27-31, 35 (in Chinese)

14. Chen SG, Zhao Y and Zhang H (2009) Analysis of large rock deformation under high in situ stress. 9th International conference on analysis of discontinuous deformation "New Developments And Applications", Singapore, 25-27 November 2012

15. Ma H, Li J, Zuo Q et al. (2012) Micro bench method for tunneling with fine blasting and its engineering application. Chin J Railway Engineering Society, 1:57-61, 82 (in Chinese) 